\title{
Modeling and Control of a Power-Split Hybrid Propulsion System
}

\author{
$1^{\text {st }}$ Davide Tebaldi \\ DIEF - University of Modena and Reggio Emilia \\ Via Pietro Vivarelli 10 Int. 1, 41100, Modena, Italy \\ davide.tebaldi@unimore.it
}

\author{
$2^{\text {nd }}$ Roberto Zanasi \\ DIEF - University of Modena and Reggio Emilia \\ Via Pietro Vivarelli 10 Int. 1, 41100, Modena, Italy \\ roberto.zanasi@unimore.it
}

\begin{abstract}
In this paper, the Power-Oriented Graphs (POG) technique is used to model a Hybrid Propulsion System for driving an agricultural tool. The main elements present in the system are: an ICE (Internal Combustion Engine), two PMSMs (Permanent Magnet Synchronous Electric Motors) equipped with two inverters in order to be properly driven, a planetary gear, an energy storage device and an agricultural tool. Based on the system dynamic model, a dedicated control strategy has been developed allowing to efficiently control the system by reducing the ICE specific consumption as much as possible. Simulation results showing the operation of the control strategy are finally reported and commented in detail.
\end{abstract}

\section{INTRODUCTION}

The interest toward hybrid propulsion systems has been largely increasing in the last years, since they provide a very large variety of advantages over non-hybrid, traditional propulsion systems, including fuel consumption reduction leading to an improvement of the environmental sustainability. The latter two issues are very important points nowadays for all the companies working in the agricultural and automotive fields. In order to draw the maximum benefits from hybrid architectures, the need of providing very accurate and effective control strategies is absolutely imperative; the starting point for this is always a well-developed and accurate model of the entire system.

This paper describes the modeling and the developed dedicated control strategy of a Power-Split hybrid architecture. The main element upon which everything is based is the powersplit device, whose role is played by a planetary gear. Such power-split topologies using a planetary gear as a power-split device, i.e. a three-ports element, are typically characterized by the presence of three contemporary power sources, namely an ICE and two electric machines. The load is represented by a shaft, to which the system to be driven is connected. The latter can be the transmission system of the vehicle or, in the case study being subject of this paper, an agricultural tool. The three power sources are clearly not independent of each other: the ICE is the main power source, i.e. the element which is responsible for generating the mechanical power that is going to be split along two different paths, see [1]. A defined fraction of the ICE power will contribute to the power level demanded by the load, whereas the remaining fraction will recharge the energy storage device through one of the electric machines. Consequently, the remaining fraction

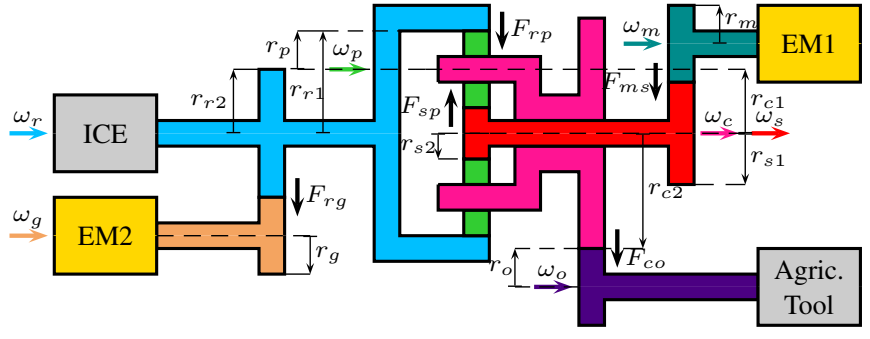

Fig. 1. Structure of the considered Power-Split hybrid propulsion system.

of power demanded by the load will be provided by the other electric machine, working in this case as a motor. One can evince that these architectures are always characterized by the coexistence of two different power paths reaching the load: a mechanical one, through which a fraction of the ICE power is delivered to the load, and an electrical one, through which the remaining part of the power demanded by the load is supplied by the electric machine working as a motor.

The electrical path represents a "noble" power path, which is generally less efficient because of the larger number of elements crossing it. Nevertheless, the presence of this alternative power path is what brings the main advantage of an architecture like this: the potentiality of making the ICE work in the most efficient operating regions for as long as possible. This can be evinced by making two observations:

- Thanks to the planetary gear, the ICE speed is decoupled from the load speed, therefore it can be chosen properly as a function of the power demand;

- Whatever torque disturbance is coming from the load, it is going to be compensated by the electric machine which is closer to the load, making it possible to choose the most proper ICE torque to minimize the specific consumption according to the current ICE speed.

The considered hybrid system shows many different configurations depending on which node of the planetary gear the different power sources are connected to, and on the type of planetary gear as well. In [2] and [3], the authors focus on the modeling of the hybrid system of a commercially available vehicle, where the employed planetary gear is in the following configuration: composed of a sun gear, a carrier gear and a ring gear. The sun gear is connected to one of the electric machines, 


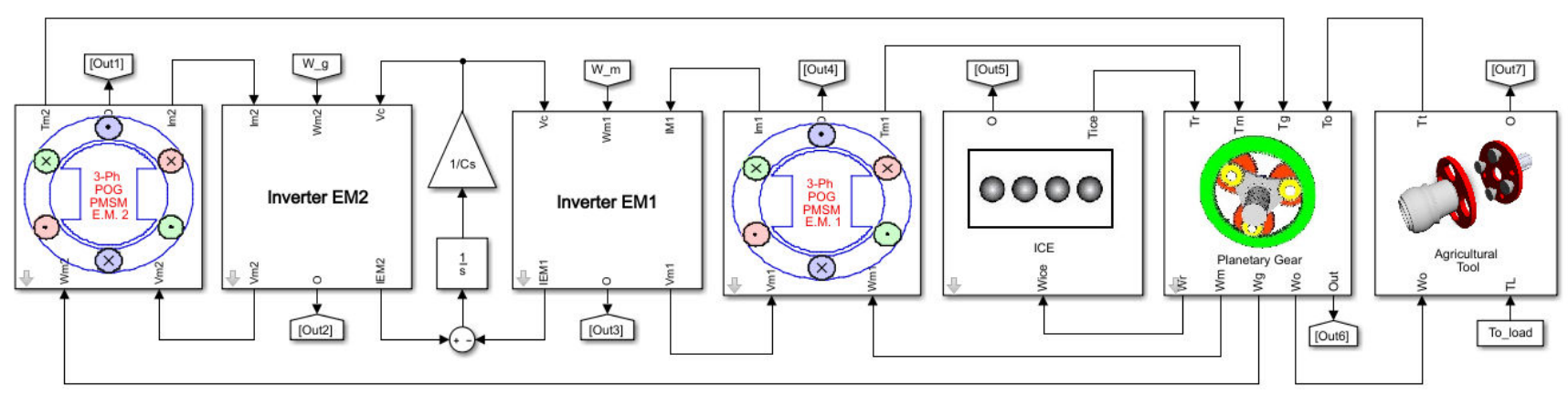

Fig. 2. Simulink block scheme of the considered Power-Split hybrid propulsion system.

the carrier gear is connected to the ICE and the ring gear is connected to the transmission system of the vehicle. The other electric machine is connected to the ring in order to perform energy recovery.

The topology presented in this paper, see Fig. 1, exploits a different configuration, where the ICE is connected to the ring gear, one of the electric machines is connected to the ring gear through an intermediate reduction ratio, the other electric machine is connected to the sun gear through an intermediate reduction ratio and the agricultural tool is connected to the carrier through an additional intermediate reduction ratio. In principle, both electric machines can work as a motor or as a generator depending on the system conditions; for this reason, reference to the electric machine closer to the load will be made as "EM1" and reference to the electric machine closer to the ICE will be made as "EM2".

The most interesting and important issue regarding these architectures is the power management problem, that is how the three power sources are controlled in order to minimize the ICE fuel consumption, thus improving the environmental sustainability as well. This problem has been addressed by the authors in [4] and [5].

In this paper, we propose a control strategy for solving the power management problem which is based on the derivation of a minimum specific consumption path for the ICE. Such strategy allows to ensure a low-consumption control policy for the ICE, allows the compensation of the torque disturbances coming from the load and guarantees that the power demand from the load is satisfied.

The paper is organized as follows: Sec. II describes the modeling of the considered hybrid system shown in Fig. 1, giving particular focus to the planetary gear, i.e. the powersplit device. Sec. III describes the idea and the implementation of the strategy which controls the three power sources to satisfy the demands. Sec. IV shows the results of a simulation showing the effectiveness of the presented control strategy. Finally, the conclusions of this work are presented in Sec. V.

\section{Modeling OF THE Hybrid System}

The power-split hybrid architecture which is subject of study in this paper is reported in Fig. 1. The corresponding Simulink block scheme is shown in Fig. 2. The elements composing the hybrid architecture in Fig. 2 are, from left to right, the electric machine denoted by "EM2", the inverter controlling this latter element, the energy storage device (i.e. the supercapacitor $C_{s}$ ), the inverter controlling the electric machine EM1, the electric machine EM1, the ICE, the planetary gear acting as a powersplit device and, finally, the agricultural tool representing the load of the system.

The electric machines used in this architecture are PMSMs and have been modeled by using the POG technique, [6]- [7]. Two industrial PMSMs have been used for this project; the actual parameters values used in the machines models have been estimated from the machines datasheet by applying an estimation procedure based on a least square algorithm, as described by the authors in [8].

The inverters employed to control the two PMSMs apply a vectorial control based on the POG state space equations, see [9].

The energy storage device used in the proposed hybrid architecture is a supercapacitor; its simple dynamic model has been obtained by using the POG modeling technique.

The internal combustion engine has been modeled within the subsystem named "ICE" in Fig. 2 and the control strategy develop for this element is described in detail in Sec III-A.

The shaft of the agricultural tool has been modeled as an inertia equipped with a linear friction coefficient accounting for the friction losses; an external torque profile can then be applied in order to simulate the presence of a disturbance coming from a real case scenario on the field.

Finally, the dynamic model of the key element, that is the planetary gear system, is described in the following Sec. II-A.

\section{A. Planetary gear modeling}

The planetary gear shown in Fig. 1 has been modeled by using the systematic and effective approach exploiting the POG technique described by the authors in [10].

The POG state space equations of the considered planetary gear are graphically shown in Fig. 3 and mathematically described by the following POG state space model:

$$
\left\{\begin{array}{rl}
\mathbf{L} \dot{\mathbf{x}} & =\mathbf{A} \mathbf{x}+\mathbf{B} \mathbf{u} \\
\mathbf{y} & =\mathbf{B}^{\mathrm{T}} \mathbf{x}
\end{array}, \quad \mathbf{x}=\left[\begin{array}{l}
\boldsymbol{\omega} \\
\mathbf{F}
\end{array}\right], \quad \mathbf{B}=\left[\begin{array}{l}
\mathbf{I} \\
\mathbf{0}
\end{array}\right]\right.
$$

where $\mathbf{x}, \mathbf{u}$ and $\mathbf{y}$ are the state, the input and the output vectors, respectively, $\mathbf{B}$ is the input matrix and $\mathbf{I}$ is an identity matrix 


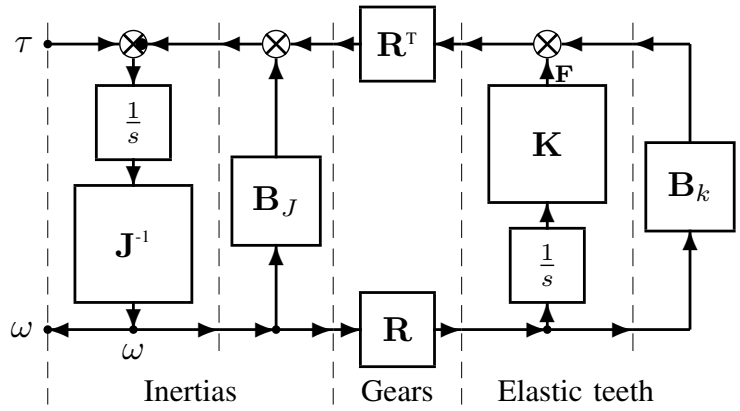

Fig. 3. Compact POG scheme of the considered planetary gear.

of proper dimension. The energy and power matrices $\mathbf{L}$ and A have the following structure:

$$
\mathbf{L}=\left[\begin{array}{cc}
\mathbf{J} & \mathbf{0} \\
\mathbf{0} & \mathbf{K}^{-1}
\end{array}\right], \quad \mathbf{A}=\left[\begin{array}{cc}
-\mathbf{B}_{J}-\mathbf{R}^{\mathrm{T}} \mathbf{B}_{k} \mathbf{R} & -\mathbf{R}^{\mathrm{T}} \\
\mathbf{R} & \mathbf{0}
\end{array}\right]
$$

The velocity vector $\boldsymbol{\omega}$, the input torque vector $\mathbf{u}=\boldsymbol{\tau}$ and the force vector $\mathbf{F}$ are defined as follows:

$$
\begin{aligned}
& \boldsymbol{\omega}=\left[\begin{array}{lllllll}
w_{r} & w_{m} & w_{s} & w_{c} & w_{p} & w_{g} & w_{o}
\end{array}\right]^{T} \\
& \boldsymbol{\tau}=\left[T_{r} T_{m} T_{s} T_{c} T_{p} T_{g} T_{o}\right]^{T}
\end{aligned}
$$

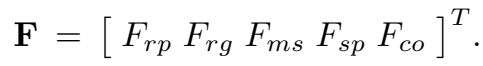

The inertia matrix $\mathbf{J}$, the inertia friction matrix $\mathbf{B}_{J}$, the stiffness matrix $\mathbf{K}$ and the stiffness friction matrix $\mathbf{B}_{K}$ are:

$$
\begin{aligned}
\mathbf{J} & =\operatorname{diag}\left(J_{r}, J_{m}, J_{s}, J_{c}, J_{p}, J_{g}, J_{o}\right), \\
\mathbf{B}_{J} & =\operatorname{diag}\left(b_{r}, b_{m}, b_{s}, b_{c}, b_{p}, b_{g}, b_{o}\right), \\
\mathbf{K} & =\operatorname{diag}\left(K_{r p}, K_{r g}, K_{m s}, K_{s p}, K_{c o}\right), \\
\mathbf{B}_{K} & =\operatorname{diag}\left(b_{r p}, b_{r g}, b_{m s}, b_{s p}, b_{c o}\right) .
\end{aligned}
$$

The radius matrix $\mathbf{R}$ has the following form:

$$
\mathbf{R}=\left[\begin{array}{ccccccc}
r_{r 1} & 0 & 0 & -r_{c 1} & -r_{p} & 0 & 0 \\
-r_{r 2} & 0 & 0 & 0 & 0 & -r_{g} & 0 \\
0 & -r_{m} & -r_{s 1} & 0 & 0 & 0 & 0 \\
0 & 0 & r_{s 2} & -r_{c 1} & r_{p} & 0 & 0 \\
0 & 0 & 0 & -r_{c 2} & 0 & 0 & -r_{o}
\end{array}\right]
$$

The radii present within matrix $\mathbf{R}$ are defined in Fig. 1 and constrained as follows: $r_{c 1}=r_{p}+r_{s 2}$ and $r_{r 1}=2 r_{p}+r_{s 2}$.

When $\mathbf{K} \rightarrow \infty$, from the state space model (1) one obtains:

$$
\mathbf{R} \omega=0 \quad \Leftrightarrow \quad\left\{\begin{array}{r}
r_{r 1} \omega_{r}-r_{p} \omega_{p}-r_{c 1} \omega_{c}=0 \\
-r_{g} \omega_{g}-r_{r 2} \omega_{r}=0 \\
-r_{m} \omega_{m}-r_{s 1} \omega_{s}=0 \\
r_{p} \omega_{p}-r_{c 1} \omega_{c}+r_{s 2} \omega_{s}=0 \\
-r_{c 2} \omega_{c}-r_{o} \omega_{o}=0
\end{array}\right.
$$

From (2), the state vector $\mathbf{x}$ can be expressed as a function of the two angular speeds $\omega_{c}$ and $\omega_{r}$ :

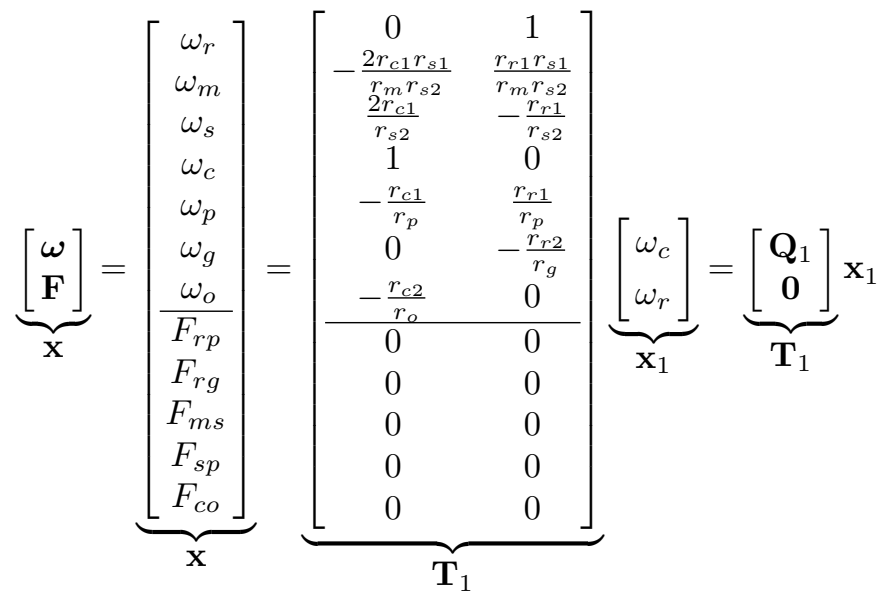

By applying the congruent transformation $\mathbf{x}=\mathbf{T}_{1} \mathbf{x}_{1}$ to system (1), one obtains the following reduced system $\mathbf{L}_{1} \dot{\mathbf{x}}_{1}=$ $\mathbf{A}_{1} \mathbf{x}_{1}+\mathbf{B}_{1} \mathbf{u}$ :

$$
\underbrace{\left[\begin{array}{ll}
J_{1} & J_{3} \\
J_{3} & J_{2}
\end{array}\right]}_{\mathbf{L}_{1}} \underbrace{\left[\begin{array}{c}
\dot{\omega}_{c} \\
\dot{\omega}_{r}
\end{array}\right]}_{\dot{\mathbf{x}}_{1}}=\underbrace{\left[\begin{array}{ll}
b_{1} & b_{3} \\
b_{3} & b_{2}
\end{array}\right]}_{\mathbf{A}_{1}} \underbrace{\left[\begin{array}{c}
\omega_{c} \\
\omega_{r}
\end{array}\right]}_{\mathbf{x}_{1}}+\underbrace{\mathbf{Q}_{1}^{\mathrm{T}}}_{\mathbf{B}_{1}} \underbrace{\tau}_{\mathbf{u}}
$$

where $\mathbf{L}_{1}=\mathbf{T}_{1}^{\mathrm{T}} \mathbf{L} \mathbf{T}_{1}, \mathbf{A}_{1}=\mathbf{T}_{1}^{\mathrm{T}} \mathbf{A} \mathbf{T}_{1}$ and $\mathbf{B}_{1}=\mathbf{T}_{1}^{\mathrm{T}} \mathbf{B}$, see [10]. The rigid planetary gear model (3) is the one used in Sec. IV for the simulation of the presented hybrid propulsion system.

\section{Control Strategy}

This section provides an answer to the following question: "how is the power management problem addressed?". The power demand coming from the load is satisfied thanks to the joint work of the three power sources present within the considered architecture: the ICE, EM1 and EM2, see Fig. 1 and Fig. 2. Therefore, the answer to the above question can be provided by describing the control applied to each of the three different power sources and the interactions among them.

\section{A. Control of the ICE}

The presence of a power-split device such as a planetary gear allows to decouple the ICE speed from the load speed, thus allowing to choose the most suitable ICE speed according to the ICE power demand. A speed control is therefore applied to the ICE, implemented by a properly designed PID (Proportional-Integral-Derivative) regulator.

The objective of the control strategy is to make the ICE work in the most efficient operating regions for as long as possible to minimize the fuel consumption. For this endothermic power source, the most efficient operating regions correspond to those where the ICE specific consumption is minimized. A piece of information which is typically made available by the provider is the ICE specific consumption map reported on a two-dimensional operating plane $\left(\omega_{i c e}, \tau_{i c e}\right)$ having the ICE speed $\omega_{i c e}$ on the $\mathrm{x}$ axis and the ICE torque $\tau_{i c e}$ on the y-axis. In order to minimize the consumption, it is useful to determine 


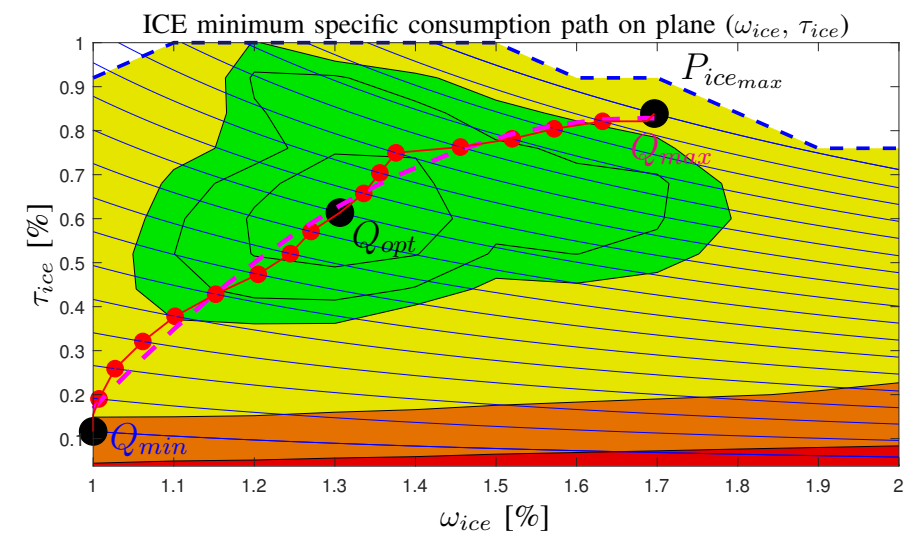

Fig. 4. ICE minimum specific consumption path on the operating plane $\left(\omega_{\text {ice }}, \tau_{\text {ice }}\right)$.

a minimum specific consumption path on the operating plane $\left(\omega_{\text {ice }}, \tau_{\text {ice }}\right)$ by following these steps:

1) Trace the constant power curves from zero to the maximum ICE power $P_{i c e_{\max }}$ on plane $\left(\omega_{i c e}, \tau_{i c e}\right)$, see the blue lines in Fig. 4;

2) By using the ICE specific consumption map, determine the most suitable operating point on each constant power curve, that is the point minimizing the ICE specific consumption;

3) Approximate the obtained suitable operating points by using a proper smooth mathematical function, in order to ensure smooth transitions from an operating point to another when the power demand changes;

In the current case, the different constant power curves have been traced with a precision $\Delta_{P_{i c e}}=10 \mathrm{~kW}$. A dedicated Matlab function has been created, whose purpose is to perform the second step of the procedure on plane $\left(\omega_{i c e}, \tau_{i c e}\right)$. For the employed ICE, the computed points on each constant power curve are marked by the red and black dots reported in Fig. 4. In order to derive a smoother characteristic, the path drawn in red in Fig. 4 has been approximated by using the following second order polynomial function:

$$
\tau_{i c e}=a_{0}+\omega_{i c e} a_{1}+\omega_{i c e}^{2} a_{2}
$$

where

$$
a_{0}=-4.01 \cdot 10^{3}, \quad a_{1}=57.41, \quad a_{2}=-0.16 .
$$

The smooth path (4) represents the chosen minimum specific consumption path on the ICE specific consumption map and is reported in magenta in Fig. 4.

Once the minimum specific consumption path is computed, the most suitable point on such path is determined as a nonlinear function of the voltage $V_{c}$ across the supercapacitor, which gives an indication about the state of charge of the supercapacitor itself. The adopted non-linear function generating

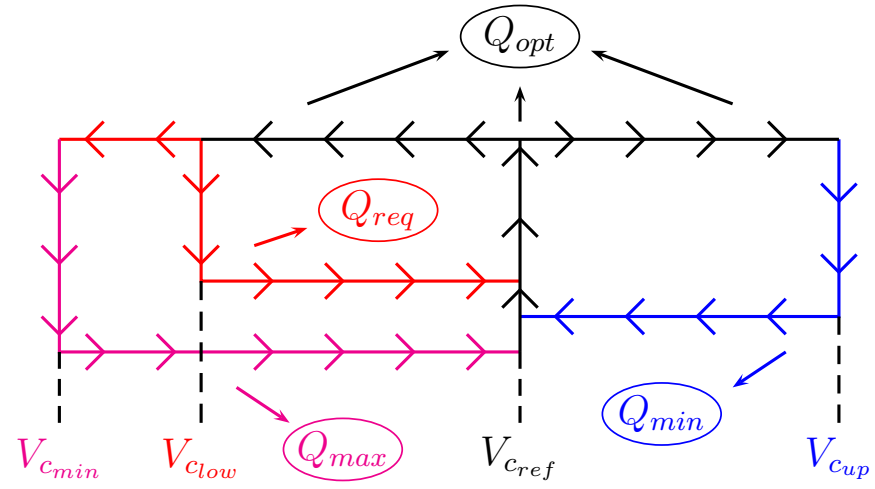

Fig. 5. Hysteresis control of the ICE.

an hysteresis control for the ICE is graphically described in Fig. 5 and logically described as follows:

$$
\left(\omega_{\text {ice }_{d e s}}, \tau_{\text {ice }_{\text {des }}}\right)=\left\{\begin{array}{lll}
Q_{o p t} & \text { if } & V_{c_{\text {low }}}<V_{c}<V_{c_{u p}} \\
Q_{r e q} & \text { if } & V_{c} \leq V_{c_{\text {low }}} \text { until } V_{c}=V_{c_{r e f}} \\
Q_{\max } & \text { if } & V_{c} \leq V_{c_{m i n}} \text { until } V_{c}=V_{c_{r e f}} \\
Q_{\text {min }} & \text { if } & V_{c} \geq V_{c_{u p}} \text { until } V_{c}=V_{c_{r e f}}
\end{array}\right.
$$

where $Q_{o p t}, Q_{\text {req }}, Q_{\max }$ and $Q_{\min }$ are defined as follows:

$$
\begin{gathered}
Q_{o p t}=\left(\omega_{i c e_{o p t}}, \tau_{i c e_{o p t}}\right), \quad Q_{r e q}=\left(\omega_{i c e_{r e q}}, \tau_{i c e_{r e q}}\right), \\
Q_{\max }=\left(\omega_{i c e_{\max }}, \tau_{i c e_{\max }}\right), \quad Q_{\min }=\left(\omega_{i c e_{\min }}, \tau_{i c e_{\min }}\right)
\end{gathered}
$$

The operating points $Q_{o p t}, Q_{\max }$ and $Q_{\min }$ are shown in Fig. 4 and belong to the minimum specific consumption path. Voltages $V_{c_{\text {min }}}, V_{c_{l o w}}$ and $V_{c_{u p}}$ are the minimum, the lower and the upper thresholds for the supercapacitor voltage $V_{c}$. These voltages satisfy the following inequalities: $V_{c_{m i n}}<$ $V_{c_{\text {low }}}<V_{c_{\text {up }}}$. The reference voltage $V_{c_{r e f}}=\left(V_{c_{\text {up }}}+V_{c_{\text {low }}}\right) / 2$ is the mean value between the two voltages $V_{c_{\text {up }}}$ and $V_{c_{l o w}}$. As a safety measure, whenever the condition $V_{c} \leq V_{c_{\text {min }}}$ is verified, the desired ICE operating point is set as follows $\left(\omega_{\text {ice }_{\text {des }}}, \tau_{\text {ice }}{ }_{\text {des }}\right)=Q_{\text {max }}$, until $V_{c}=V_{c_{\text {ref }}}$ is once again verified, in order to avoid excessive discharge of the supercapacitor because of possible peaks from the load external disturbance torque.

The ICE operating points $Q_{o p t}, Q_{\max }$ and $Q_{\min }$ are chosen on the minimum specific consumption path as described in the following. The optimal operating point $Q_{o p t}$ is the closest to the minimum consumption point in the center of the inner green area of the specific consumption map shown in Fig. 4. The minimum operating point $Q_{\min }$ is the minimum point on the minimum specific consumption path: $\omega_{\text {ice }_{\text {min }}}=\min \left(\omega_{i c e}\right)$ and $\tau_{i c e_{\min }}=\min \left(\tau_{i c e}\right)$. The maximum operating point $Q_{\max }$ is the maximum point on the minimum specific consumption path: $\omega_{\text {ice }_{\max }}=\max \left(\omega_{\text {ice }}\right)$ and $\tau_{\text {ice } \max }=\max \left(\tau_{\text {ice }}\right)$.

The use of a required operating point $Q_{r e q}$, instead of using the maximum operating point $Q_{\max }$, when $V_{c} \leq V_{c_{l o w}}$ allows to keep the operating point as close to the green 
area in Fig. 4 as possible, unless it is strictly necessary to move it, that is if condition $V_{c} \leq V_{c_{\min }}$ occurs. The philosophy behind this reasoning is indeed the one specified at the beginning of this section: the minimization of the ICE specific consumption whenever possible. The required operating point $Q_{r e q}=\left(\omega_{\text {ice }_{\text {req }}}, \tau_{\text {ice }_{\text {req }}}\right)$ when $V_{c} \leq V_{c_{\text {low }}}$ is computed as follows:

$$
\begin{aligned}
& \text { 1) } \Delta_{P}=\frac{\Delta_{E}}{\Delta_{T}}=\frac{\frac{1}{2} C_{s} V_{c_{r e f}}^{2}-\frac{1}{2} C_{s} V_{c_{\text {low }}}^{2}}{\Delta_{T}} \\
& \text { 2) } P_{i c e_{r e q}}=P_{i c e_{o p t}}+\Delta_{P}=\omega_{i c e_{o p t}} \tau_{i c e_{o p t}}+\Delta_{P} \\
& \text { 3) } \omega_{i c e_{r e q}}=K P_{i c e_{r e q}} \\
& \text { 4) } \tau_{i c e_{r e q}}=a_{0}+\omega_{i c e_{r e q}} a_{1}+\omega_{i c e_{r e q}}^{2} a_{2}
\end{aligned}
$$

where $\Delta_{P}$ is the ICE required additional power, $\Delta_{T}=$ $t_{\text {low }}-t_{\text {ref }}$ is the time interval between the instant $t_{\text {low }}$ when $V_{c}=V_{c_{\text {low }}}$ and the instant $t_{\text {ref }}$ when $V_{c}=V_{c_{\text {ref }}}, K$ is a proportional coefficient properly chosen, and the polynomial used in step 4) is the one defined in (4). The increasing of the ICE power by the required $\Delta_{P}$ has two consequences:

1) The reduction of the power that EM1 provides to the load, thus the reduction of the power absorbed from the supercapacitor;

2) The increase of the power flowing through EM2 and entering the supercapacitor.

Both consequences contribute to the recharge of the supercapacitor itself. In addition, note that the relation reported in step 3), where the required ICE speed $\omega_{i c e_{r e q}}$ is chosen proportional to the ICE demanded power $P_{i c e_{r e q}}$, well highlights the speed control applied to the ICE.

An important observation has to be made: the dimensioning of the supercapacitor affects the frequency of variation of the ICE operating point. This can be evinced by looking at the relation $\Delta_{P}=\Delta_{E} / \Delta_{T}$ appearing in the first step of the computation of the ICE required operating point when $V_{c} \leq V_{c_{\text {low }}}$ reported above. The quantity $\Delta_{E}$, representing the difference between the energy stored within the supercapacitor when $V_{c}=V_{c_{r e f}}$ and the energy stored within the supercapacitor when $V_{c}=V_{c_{l o w}}$, is directly proportional to the capacitance value $C_{s}$. By assuming that the difference between the power exiting and entering the supercapacitor, $P_{\text {out }}-P_{i n}$, is positive and constant over a certain time interval, the supercapacitor voltage $V_{c}$ starts decreasing with a constant slope, and the time it takes for $V_{c}$ to decrease by a certain $\Delta_{V_{c}}$ is directly proportional to the capacitance $C_{s}$. This means that the capacitance value $C_{s}$ has to be chosen properly, according to these considerations, as a function of the average power absorbed by the load for a given application, in order not to cause excessive variations of the ICE operating point, thus improving the end user driving/working experience.

\section{B. Control of Electric Machine EM2}

The ICE control strategy described in Sec. III-A clearly shows the presence of a speed control applied to the ICE. Since both the ICE and EM2 are connected to the ring of the planetary gear, the latter through an additional reduction ratio, and a speed control is applied to the ICE, it is straightforward that the only remaining power variable of EM2 which can be controlled is the generated torque.

The main goal of the torque control applied to EM2 is to ensure that the ICE torque is always equal to the desired torque $\tau_{\text {ice }}$ pros provided by the hysteresis control described in (5) as a function of the desired ICE speed $\omega_{\text {ice des }}$, in order to confine the ICE operating point on the minimum specific consumption path. Therefore, the desired torque $\tau_{m 2_{d e s}}$ for the Electric Machine EM2 is chosen as follows:

$$
\tau_{m 2_{\text {des }}}=K_{m 2} \Delta_{\tau_{\text {ice }}}=K_{m 2}\left(\tau_{\text {ice des }}-\tau_{\text {ice }}\right)
$$

where $K_{m 2}$ is a properly chosen proportional coefficient. The desired torque $\tau_{m 2_{d e s}}$ is then demanded to EM2 by means of an inverter, which has been inserted within subsystem "Inverter EM2" in Fig. 2.

\section{Control of Electric Machine EMI}

The description of the controls applied to the ICE and to EM2, reported in Sec. III-A and in Sec. III-B respectively, highlights that the ICE speed and, consequently, the speed of EM2 are chosen as a function of the power demand to the ICE and are decoupled from the load speed thanks to the presence of the planetary gear. From the latter observations, it is straightforward to state that the element being responsible to make the load follow the desired speed profile $\omega_{o_{d e s}}$ is EM1. By using relations (2), one can easily verify that the desired speed $\omega_{m 1_{\text {des }}}$ for EM1 can be expressed as follows:

$$
\omega_{m 1_{d e s}}=-\frac{r_{s 1}}{r_{m}}\left[\frac{-\frac{2 r_{c 1} r_{o}}{r_{c 2}} \omega_{o_{d e s}}-r_{r 1} \omega_{i c e_{d e s}}}{r_{s 2}}\right]
$$

as a function of the desired ICE speed $\omega_{i c e_{d e s}}$ and the desired load speed $\omega_{o_{\text {des }}}$, respectively. The radii $r_{s 1}, r_{m}, r_{c 1}, r_{o}, r_{c 2}$, $r_{r 1}$ and $r_{s 2}$ in (6) are defined in Fig. 1.

A PID regulator is then employed to convert the speed tracking error $\omega_{m 1_{d e s}}-\omega_{m 1}$ of EM1 into a desired torque $\tau_{m 1_{\text {des }}}$. The element being responsible to apply the speed control to EM1 in order to make the load follow the desired speed profile $\omega_{\text {odes }}$ is the inverter inserted within subsystem "Inverter EM1" in Fig. 2.

\section{Simulation RESUlts}

The architecture shown in Fig. 2 has been tested in simulation referring to an industrial case study: the objective of the simulation is to keep the angular speed $\omega_{0}$ of the load shaft equal to a constant value $\omega_{o_{\text {des }}}$ in presence of an external disturbance, namely a resistive torque $\tau_{\text {oload }_{\text {lod }}}$ applied to the load. The obtained simulation results are shown in Fig. 6, Fig. 7, Fig. 8, Fig. 9 and Fig. 10. The plots reported in the figures have been normalized for trade secret reasons, 

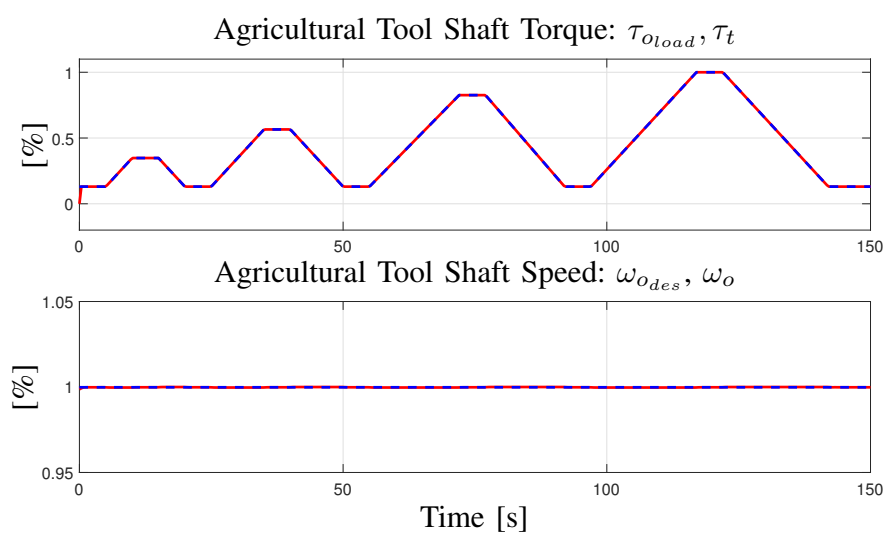

Fig. 6. Resistive and motive agricultural tool shaft torque; Desired and actual agricultural tool shaft speed.
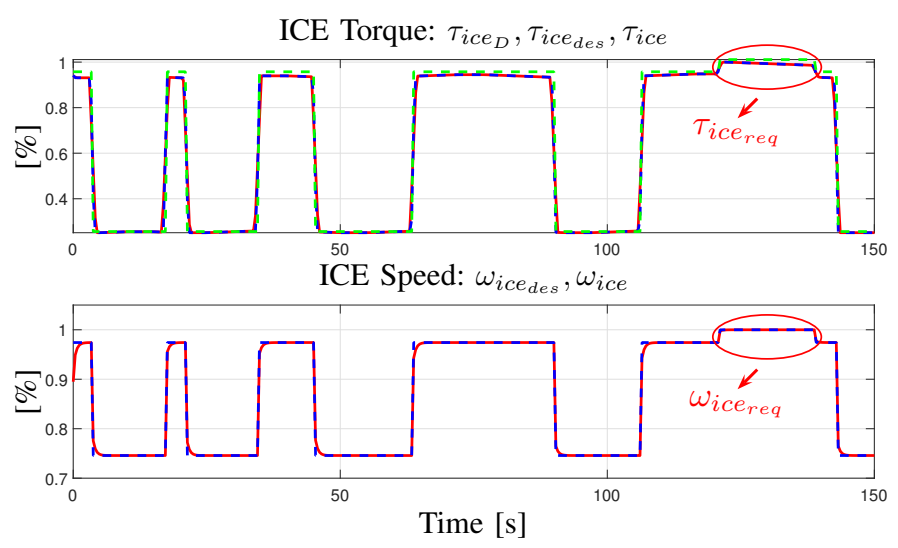

Fig. 7. Demanded, desired and actual ICE torque; Desired and actual ICE speed.

however the reader can still appreciate the results from the characteristics behavior.

ICE Operating Points

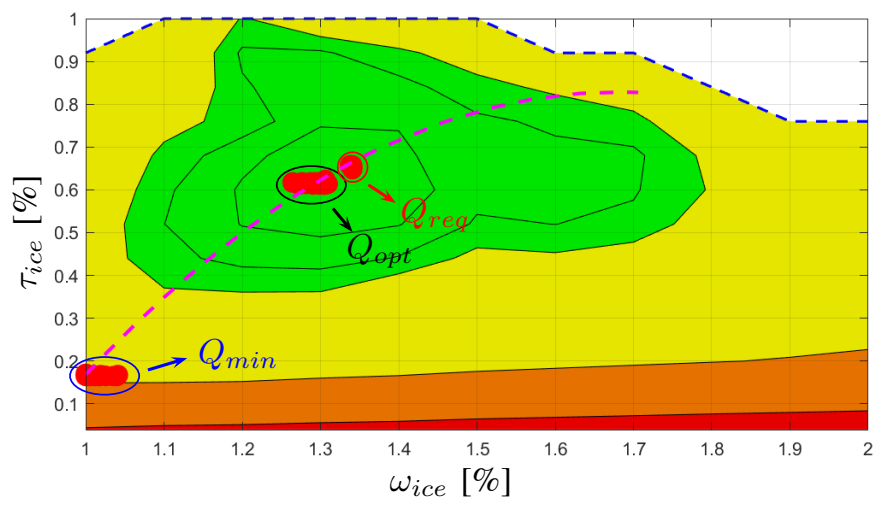

Fig. 8. ICE operating points and ICE minimum specific consumption path on the specific consumption map.

The upper subplot in Fig. 6 shows the external torque disturbance $\tau_{\text {oload }}$ applied to the load (blue dashed curve) and the torque $\tau_{t}$ made available by the joint contribution of the ICE and EM1 (red curve). The lower subplot in Fig. 6 shows the desired load speed $\omega_{o_{\text {des }}}$ (blue dashed line) and the actual

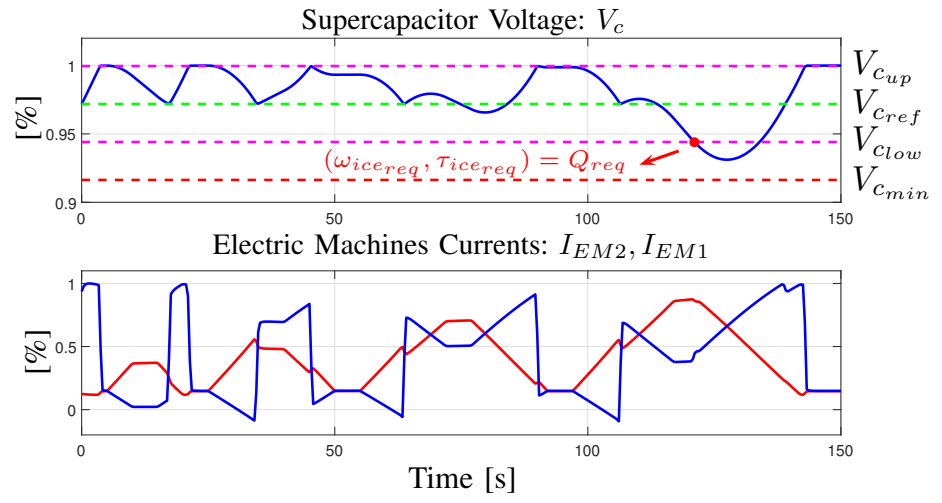

Fig. 9. Voltage across the supercapacitor; Currents from the inverters.
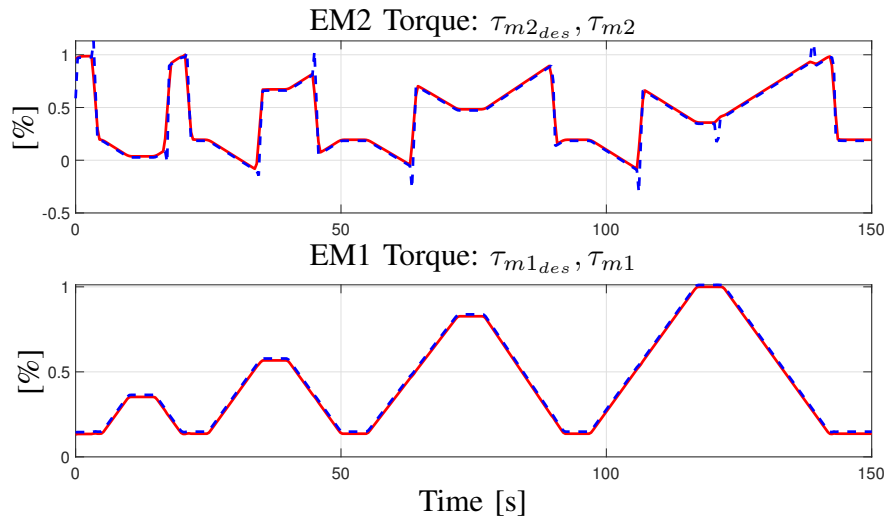

Fig. 10. Desired and actual EM2 torques; Desired and actual EM1 torques.

load speed $\omega_{o}$ (red line). The good tracking of the desired signals shows the effectiveness of the speed control applied to EM1.

The upper subplot in Fig. 7 shows: the torque $\tau_{i c e_{D}}$ demanded by the ICE speed control (blue dashed characteristic), the ICE desired torque $\tau_{\text {ice }}$ (green dashed line), and the ICE actual torque $\tau_{i c e}$ (red characteristic). The green and red characteristics are very close, denoting the effectiveness of the torque control applied to EM2. The lower subplot in Fig. 7 shows the ICE desired speed $\omega_{\text {ice }}$ (blue dashed curve) and the ICE actual speed $\omega_{i c e}$ (red curve). The good tracking of the desired signal shows the effectiveness of the speed control applied to the ICE.

The ICE operating points on the ICE consumption map are shown in red in Fig. 8. This figure clearly shows that the ICE operating points are always very close to the minimum specific consumption path (dashed magenta curve), denoting the effectiveness of the proposed control strategy. In particular, in this simulation there are three different zones along the minimum specific consumption path where the ICE operating points are grouped: 1) the minimum power zone corresponding to minimum operating point $Q_{\min }$ (zone delimited by the blue ellipse in Fig. 8, 2) the lowest consumption zone corresponding to the optimal operating point $Q_{\text {opt }}$ (zone delimited by the black ellipse in Fig. 8, and 3) the zone of the path corresponding to the required operating point $Q_{r e q}$ 
in correspondence of a time interval where $V_{c}$ needs to be increased from $V_{c} \leq V_{c_{\text {low }}}$ to $V_{c}=V_{c_{\text {ref }}}$ (zone delimited by the red circle in Fig. 8, very close to the middle of the inner green area). The time intervals in which the ICE operates in the required operating point $Q_{r e q}$ are also highlighted in Fig. 7 by two red ellipses: the one in the upper subplot highlights when $\tau_{i c e}=\tau_{i c e_{r e q}}$, the one in the lower subplot highlights when $\omega_{\text {ice }}=\omega_{\text {ice }}$ req .

The blue curve in the upper subplot of Fig. 9 shows the supercapacitor voltage $V_{c}$ and the thresholds used in the hysteresis control law (5): $V_{c_{\text {low }}}$ (lower magenta dashed line), $V_{c_{u p}}$ (upper magenta dashed line), $V_{c_{m i n}}$ (lowest red dashed line) and $V_{c_{r e f}}$ (green dashed line). The red dot present in this subplot highlights the time instant when voltage $V_{c}$ becomes lower than threshold $V_{c_{l o w}}$, in which the procedure for computing the ICE required operating point $Q_{r e q}$ on the minimum specific consumption path described in Sec. III-A is activated. Then, the speed control applied to the ICE and the torque control applied to EM2 act in order to take the ICE to the required operating point: $\left(\omega_{\text {ice }_{r e q}}, \tau_{i c e_{r e q}}\right)=Q_{r e q}$ as it can be seen from Fig. 7 and Fig. 8.

The lower subplot of Fig. 9 shows the current $I_{E M 2}$ flowing through EM2 and entering the supercapacitor (blue curve), and the current $I_{E M 1}$ exiting the supercapacitor and flowing through EM1 (red curve).

The desired and actual torques of EM1 and EM2, $\tau_{m 1_{d e s}}, \tau_{m 2_{d e s}}, \tau_{m 1}$ and $\tau_{m 2}$, are shown in Fig. 10 in blue dashed line and in red line, respectively. The desired torque $\tau_{m 2_{\text {des }}}$ has been designed to minimize the ICE torque error $\Delta_{\tau_{i c e}}$, and the desired torque $\tau_{m 1_{\text {des }}}$ has been designed to compensate for the external disturbance $\tau_{\text {oload }}$ of the load.

\section{CONCLUSIONS}

In this paper, the modeling and the control of a power-split hybrid architecture have been presented. The modeling of the hybrid system has been made by using the POG modeling technique, which allows an excellent control of the power flows within the system. Then, an effective solution for the power management problem has been presented, allowing to minimize the ICE specific fuel consumption and provide the load with a power flow which allows to follow the desired speed and to compensate for the external disturbances applied to the load shaft. The simulation results show the effectiveness of the proposed control strategy for controlling the considered hybrid propulsion system.

\section{REFERENCES}

[1] J. M. Miller, "Hybrid Electric Vehicle Propulsion System Architectures of the e-CVT Type", IEEE Trans. on Power Electronics, May. 2006.

[2] J. Liu, H. Peng, Z. Filipi, "Modeling and Analysis of the Toyota Hybrid System", Proceedings of the IEEE/ASME International Conference on Advanced Intelligent Mechatronics, Monterey, California, USA, July 24$28,2005$.

[3] J. Liu, H. Peng, "Modeling and Control of a Power-Split Hybrid Vehicle", IEEE Trans. on Control Systems Technology, Nov. 2008.

[4] J. Liu, H. Peng, "Control Optimization for a Power-Split Hybrid Vehicle", Proceedings of the American Control Conference (ACC), Minneapolis, Minnesota, USA, June 14-16, 2006.
[5] C. C. Lin, H. Peng, J. W. Grizzle, "A Stochastic Control Strategy for Hybrid Electric Vehicles", Proceedings of the American Control Conference (ACC), Boston, MA, USA, June 30 - July 2, 2004.

[6] R. Zanasi, "The Power-Oriented Graphs Technique: System modeling and basic properties", IEEE Vehicle Power and Propulsion Conference, Lille, France, September 1-3, 2010.

[7] M. Fei, R. Zanasi, F. Grossi, "Modeling of Multi-phase Permanent Magnet Synchronous Motors under Open-phase Fault Condition", IEEE International Conference on Control and Automation (ICCA), Santiago, Chile, December 19-21, 2011.

[8] R. Zanasi, D. Tebaldi, "Power Flow Efficiency of Linear and Nonlinear Physical Systems", IEEE European Control Conference (ECC), Naples, Italy, June 25-28, 2019.

[9] R. Zanasi, F. Grossi, "Vectorial Control of Multi-phase Synchronous Motors using POG Approach", 35th Annual Conference of IEEE Industrial Electronics, Porto, Portugal, November 3-5, 2009.

[10] R. Zanasi, D. Tebaldi, "Planetary Gear Modeling Using the PowerOriented Graphs Technique", IEEE European Control Conference (ECC), Naples, Italy, June 25-28, 2019. 